30th International Cosmic Ray Conference

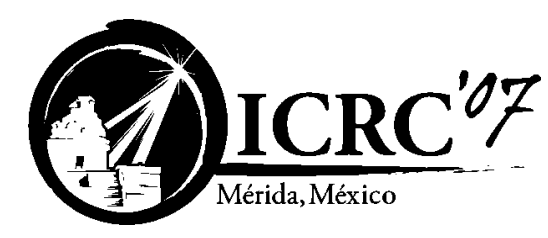

\title{
A Geant4 based engineering tool for Fresnel lenses
}

\author{
Joẽo Costa ${ }^{1}$, Mário Pimenta ${ }^{2}$, Bernardo Tomé². \\ ${ }^{1}$ Laboratoire de l'Accélérateur Linéaire, IN2P3-CNRS et Université Paris-Sud 11, Centre Scien- \\ tifique d'Orsay, B. P. 34, 91898 Orsay, Cedex, France. \\ ${ }^{2}$ Laboratório de Instrumentação e Física Experimental de Partículas, Av. Elias Garcia, 14-1, \\ 1000-149 Lisboa, Portugal. \\ bernardo@lip.pt
}

\begin{abstract}
Geant4 is a Monte Carlo radiation transport toolkit that is becoming a tool of generalized application in areas such as high-energy physics, nuclear physics, astroparticle physics, or medical physics. Geant 4 provides an optical physics process category, allowing the simulation of the production and propagation of light. Its capabilities are well tailored for the simulation of optics systems namely in cosmic-rays experiments based in the detection of Cherenkov and fluorescence light. The use of Geant4 as an engineering tool for the optics design and simulation of Fresnel lens systems is discussed through a specific example.
\end{abstract}

\section{Introduction}

The use of Fresnel lenses has been discussed in the last few years in the context of cosmicrays experiments based on the detection of the Cherenkov and fluorescence light produced by extensive air showers $[1,2,3,4]$. In the specific case of Cherenkov telescopes, the field of view (FOV) can be considerably enlarged by employing Fresnel lens based optics. In fact, the FOV of exhisting and planned Cherenkov telescopes, using reflective optics, is limited to few degrees due to the degradation of the imaging quality for off-axis angles and to the need of using small cameras at the focal plane to limit the mirror obscuration. To cover a large area of the sky, a possible alternative is to use refractive optics with the camera located behind the lens. Fresnel lenses are a viable solution due to their small thickness and lightness.

In this paper a simulation tool for Fresnel lenses using Geant4 is presented. It is shown how it can be explored to perform realistic simulations and optimizations of optics systems using these lenses.

\section{The Geant4 toolkit}

Geant4 is a toolkit to simulate the particle transport and interactions in matter, with tracking capabilities in 3D geometries of arbitrary complexity. It includes an extensive set of electromagnetic, hadronic and optics physics processes [5,6]. Optical photons are generated through scintillation, Cherenkov and transition radiation or can be explicitly emitted by a user defined light source. The tracking of optical photons includes refraction and reflection at medium boundaries, Rayleigh scattering and bulk absorption.

\section{Lens geometry implementation}

A Fresnel lens is constructed from a given lens surface by decomposing it into small pieces. Each piece of the original lens surface is translated to the plano side of the lens, thus defining a groove of the Fresnel lens.

In the present simulation the lens grooves have a conical shape, each being a frustum of a cone with a cross-section represented by a straight line. The slopes of these lines are obtained 
from the sagita equation of the original lens surface. The concentric grooves have the same radial span - constant width grooves. The geometry of a Fresnel lens is defined in Geant4 using a parameterized replication of G4Cons volumes. A lens can be described either as a monolithic lens or as a generic lens petal, covering a limited azimuthal range. A generic lens petal can be rotated around a specified axis.

\section{Simulation of a specific lens}

As a working example a $1.7 \mathrm{~m}$ diameter planoconvex Fresnel lens consisting of a central monolithic lens and two concentric rings of lens petals was simulated. This example was inspired by the innovative approach proposed by the GAW collaboration.

The lens profile, an aspheric even surface, was based on an optimization at $\lambda=320 \mathrm{~nm}$ using the OSLO[7] optics software. The focal length is $2025 \mathrm{~mm}$ and the lens material is a typical ultraviolet (UV) transmitting acrylic. Two lenses were simulated, with groove densities of 2 grooves/mm and 0.333 grooves $/ \mathrm{mm}$.

\section{Analysis of the optics}

In the lens design and optimization several parameters must be taken into consideration, namely the magnitude of the aberration, characterized by the point spread function (PSF), the lens transmittance and the telescope FOV. For the purpose of characterizing the present lens, some of its optimization parameters were varied: the density of the grooves, the thickness of the lens, and the distance from the lens to the detection plane. Some of the results obtained in this context are shown hereafter with the main purpose of illustrating the capabilities of this simulation tool. A complete optimization of a detector and the systematic characterization of its performance is out of the scope of this paper.

As a first example, the dependence of the PSF with the groove density was studied for UV light $(\lambda=320 \mathrm{~nm})$ incident parallel to the lens optical axis $\left(\theta=0^{\circ}\right)$, as shown in Fig. 1. The

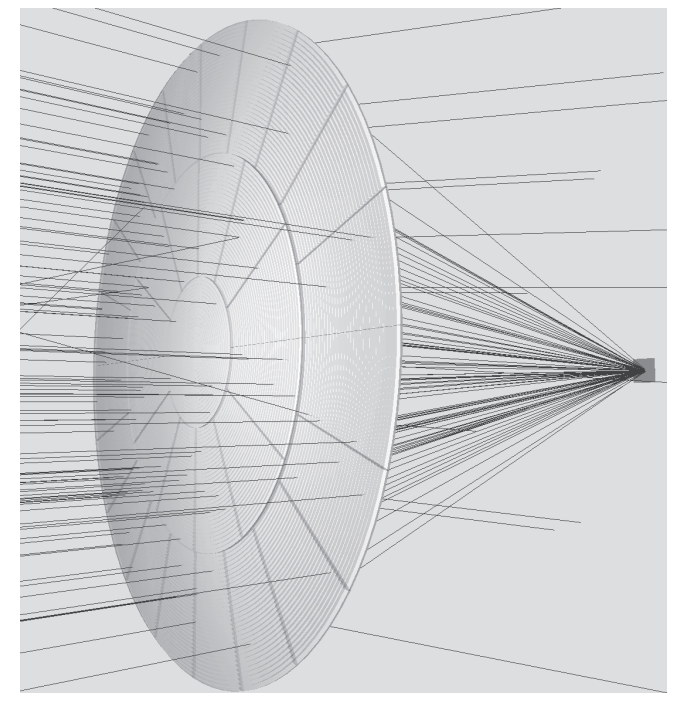

Figure 1: Visualization of the simulated Fresnel lens, illustrating the focusing of on-axis light incident from the left.

spot images at the nominal focal distance and the corresponding encircled image, the fraction of photons collected inside a circle of variable radius, are shown in Fig. 2.

The focusing was quantified by the radius of the circle at the focal plane containing $90 \%$ of the detected light $\left(\mathrm{R}_{90}\right)$. In the following studies a light beam incident on-axis was considered, yielding a PSF which is symmetric about the lens axis. However, for large incidence angles coma aberration distorts the PSF and the definition of the spot size should be modified.

The dependence of $R_{90}$ with the focal distance is shown in Fig. 3, for the two simulated lenses. For the lens with the smaller grooves the optimum distance $d_{m i n}$, the distance that minimizes $R_{90}$, is found to be close to the nominal focal length. In the case of the lens with smaller groove density the aberration can still be reduced by about $60 \%$ through the displacement of the focal plane by $5 \mathrm{~mm}$ from the nominal position. In both cases the spot size at $d_{\min }$ follows approximately the width of the grooves of the corresponding lens. This aberration is due to the flat-surface groove facets: parallel photons arriving at a given groove are all refracted by the same angle, exiting the lens in a 


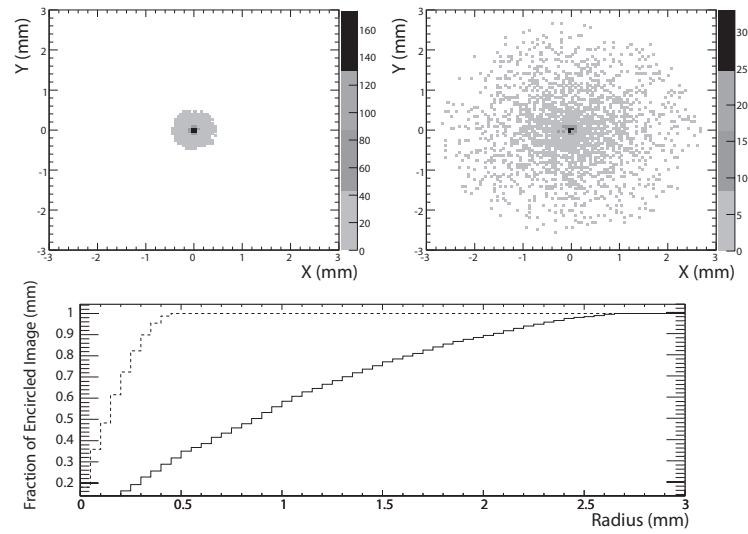

Figure 2: PSF for on-axis incidence of monochromatic light $(\lambda=320 \mathrm{~nm})$. Upper left: lens with groove density of $2 / \mathrm{mm}$. Upper right: lens with groove density of $0.333 / \mathrm{mm}$. Bottom: Encircled image vs radius for lenses with groove density of $2 / \mathrm{mm}(--)^{-}$) and $0.333 / \mathrm{mm}$ $(-)$.

parallel beam, without undergoing any focusing. This intrinsic aberration, proportional to the size of the groove, is thus a characteristic of Fresnel lenses featuring flat surface grooves. In a real cosmic ray experiment, a Cherenkov telescope will operate in an extended wavelength range and the lens optimization should take into account effects such as the chromatic aberration. The Cherenkov spectrum can be incorporated in the optimization studies thanks to the capabilities of Geant4. Effects such as the wavelength dependence of the light scattering in the atmosphere, of the light absorption in the lens material, or of the spectral sensitivity of the photo-detectors can also be easily described with Geant4.

Figure 4 shows $R_{90}$ as a function of the focal distance for monochromatic light at several wavelengths. The size of the smallest PSF is approximately the same for all wavelengths, although at different $d_{\min }$. Since the refractive index of the lens decreases with the wavelength, $d_{\min }$ increases with increasing wavelength, ranging from about $2030 \mathrm{~mm}$ for $\lambda=$ $320 \mathrm{~nm}$ to $2185 \mathrm{~mm}$ for $\lambda=600 \mathrm{~nm}$.

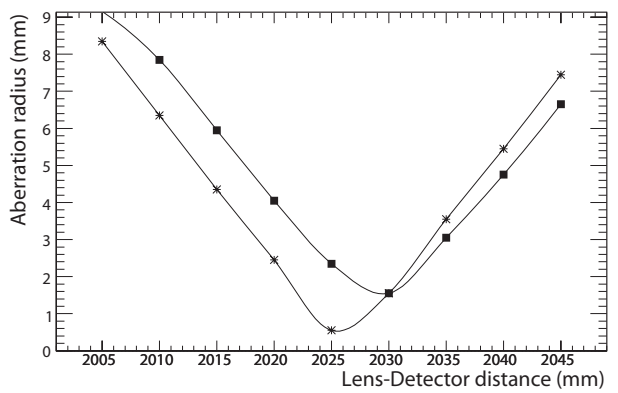

Figure 3: Aberration radius $\left(\mathrm{R}_{90}\right)$ as a function of the distance from the lens to the detector for on-axis incidence of monochromatic light $(\lambda=320 \mathrm{~nm})$. Results are shown for lenses with 2 grooves $/ \mathrm{mm}$ (*) and 0.333 grooves $/ \mathrm{mm}$ $(\boldsymbol{\square})$; the lines join the points.

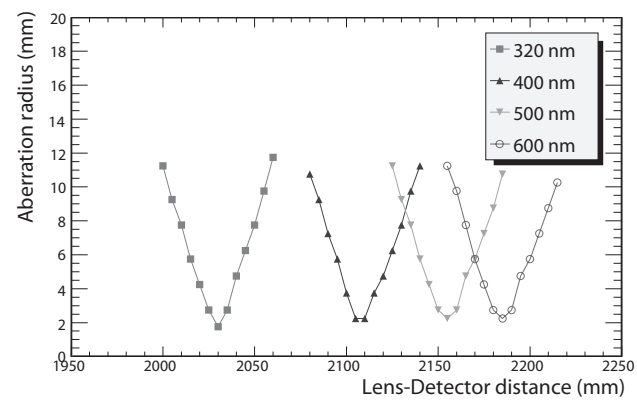

Figure 4: Aberration radius $\left(\mathrm{R}_{90}\right)$ as a function of the distance from the lens to the detector for different wavelengths.

The lens transmittance was studied as a function of the angle of incidence and of the distance from the center of the lens. Figure 5 shows the fraction of detected light as a function of the distance of the incident rays from the lens axis, for $\theta=0^{\circ}$. Two cases are shown: a lens with infinite absorption length and a lens with the typical absorption spectrum of the UV transmitting acrylic. In both cases the light loss increases by about $20 \%$ towards the edge of the lens. While the bulk absorption, of the order of $10 \%-15 \%$, is approximately constant with the radius, there is an additional effect reducing the light transmission which increases with the radius. As illustrated in Fig. 6 this is 


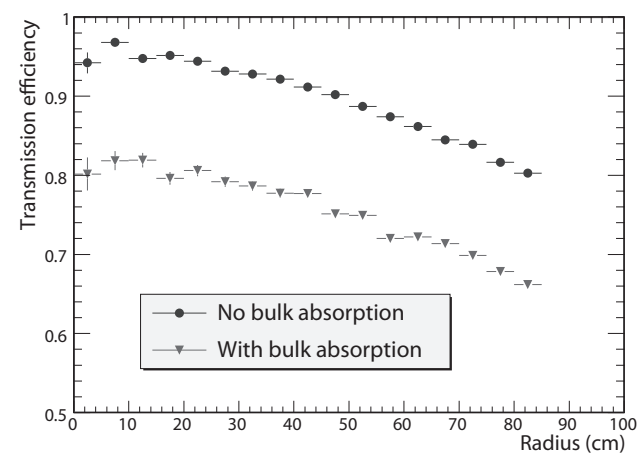

Figure 5: Transmission of light with distance from center of the lens.

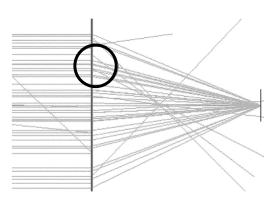

a)

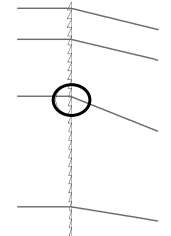

b)

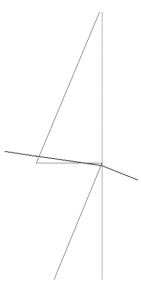

c)
Figure 6: Example of multiple refraction of photons in the lens grooves. a) Some light rays exit the lens out of focus. The encircled region in a) is shown in greater detail in b) and c), illustrating how photons impinging close to the edge of one groove can be refracted to the next groove.

due to the multiple refraction of photons which exit one groove near its border and cross an adjacent one, thereby changing their direction out of focus. As observed, this effect increases towards the lens periphery since, for constant width grooves, the groove height increases with the radius.

Besides the type of analysis described here, which can be carried out in standalone mode, it is possible to integrate the lens simulation in a complete Geant4-based detector simulation, including detector components such as light guides and photomultipliers. Such simulation can also be interfaced with an external air-shower generator or a readout electronics and signal digitization module. These possibilities are particularly useful for the simulation of fluorescence and Cherenkov telescopes for present and future cosmic-ray experiments.

\section{Summary}

In this paper an engineering tool for the optics design and simulation of Fresnel lenses using the Geant4 toolkit was presented. Performance and optimization studies of a specific lens design were described.

\section{Acknowledgements}

The authors from LIP acknowledge the support of Fundação para a Ciência e Tecnologia, Portugal.

\section{References}

[1] L. Scarsi, Il Nuovo Cimento, vol. C 24, pp. 471-482, 2001.

[2] G. Agnetta et al, Nucl. Instrum. Meth., A570, 22, 2007.

[3] P. Assis and et al.. In these proceedings, 2007.

G. Cusumano and et al.. In these proceedings, 2007.

M. C. Maccarone and et al.. In these proceedings, 2007.

[4] D. Lamb, PhD Thesis, University of Alabama in Huntsville, 1999.

[5] S. Agostinelli, J. Allison, K. Amako, J. Apostolakis, H. Araujo, P. Arce Dubois et al., Nucl. Instrum. Meth. A, A 506, 250, 2003.

[6] M. G. Pia, Nucl. Phys. B - Proc. Suppl., 125, 60, 2003.

[7] LAMBDA corporation, http://www.lambdares.com/ 